\title{
El rol del tutor en un ambiente virtual de aprendizaje para la formación continua de docentes.
}

\author{
Juan Eusebio Silva Quiroz \\ jsilva@comenius.usach.cl \\ Centro Comenius \\ Universidad de Santiago de Chile
}

\section{INTRODUCCIÓN}

Las Tecnologías de la información y comunicación (TIC) están siendo insertadas en todas las áreas de la sociedad, provocando diferentes impactos. La educación es una de estas áreas, donde las posibilidades que estas tecnologías proporcionan, pueden favorecer la introducción de aspectos innovadores en los aspectos metodológicos, relacionados con los procesos de enseñanza y aprendizaje.

La inserción de las TIC en los contextos educativos reportar beneficios para el sistema educativo en su conjunto, alumnos, docentes y la comunidad educativa en general. En el caso de los docentes, estas tecnologías ponen a disposición de éstos recursos en diferentes formatos, participar en redes de docentes, trabajar proyectos en forma colaborativa con otros centros educativos (Harasim et.al., 2000, Hepp, 2003; Crook, 1998). Una de las posibilidades emergentes derivadas de estas tecnologías instaladas en los centros educativos, es el uso de entornos virtual de aprendizaje (EVA) para apoyar la labor docente, extendiendo la clase más allá de las fronteras del aula. Además de ser útiles para formarse en forma continua en forma virtual, participando de experiencias de formación centradas en perspectivas educativas constructivista de raíces socio culturales, donde la interacción con los pares, la reflexión y el construir conocimiento en forma colaborativa son aspectos centrales.

Este artículo presenta una investigación realizada en el marco del programa de doctorado en Multimedia Educativo de la Universidad de Barcelona[1]. Aborda una experiencia concreta, de utilización de las TIC para capacitar a docentes, a través de un entorno virtual, concebido como un espacio para el aprendizaje individual y colaborativo, de contenidos matemáticos y competencias TIC. Esto último por medio de la interacción con tutores y otros alumnos, en el marco de discusiones y reflexiones. Es una investigación exploratoria, descriptiva, basada en el análisis de las intervenciones tutoriales en los espacios de trabajo del curso en estudio.

\section{MARCO TEÓRICO}

Las TIC están produciendo cambios en la formas de enseñanza y aprendizaje. En la forma en que los profesores y aprendices se relacionan con el conocimiento y las forma en que los agentes involucrados en el proceso educativo interactúan. El uso de las TIC en educación ha permitido relevar propuestas metodológicas que pedagógicamente llevan años de desarrollo (Gros, 2002). Como señala Hernández (1997) las publicaciones de revistas de tecnología educativa son de las que más páginas han dedicado al tema del constructivismo. Es tal el impacto de las TIC que se ha pasado a llamar comunicación mediada por computador a las instancias de comunicación realizadas, controladas y administradas por la tecnología computacional. Las instancias la comunicación permite mantener un flujo de información entre el profesor y los participantes, y entre estos últimos, que les facilita: poner en común ideas, compartir, reflexionar, desarrollar trabajos de carácter cooperativo y/o colaborativo, recibir retroalimentación y orientaciones por parte del tutor. 
Dentro de las herramientas comunicativas se encuentra la llamada Conferencia Mediada por Computador (CMC), una modalidad asincrónica de comunicación que permite la comunicación utilizando como base el texto escrito (Ryan et.al., 2000; Salmon, 2000; Harasim et.al., 2000; Bates, 1995). Es una herramienta que provee un ambiente electrónico para el envío y recepción de mensajes, así como para su administración. La CMC ofrece enormes posibilidades para crear entornos de aprendizaje que permiten implementar estrategias de enseñanza y aprendizajes, en las cuales la interacción social sea un elemento central. Estos entornos se centrarían en modelos constructivista de carácter sociocultural, posibilitando el trabajo colaborativo y potenciando la construcción de conocimiento en una comunidad de aprendizaje. Se promueve entonces espacios para la reflexión, accesible a toda hora, adaptables al ritmo de aprendizaje individual y por sobre todo opuestos a la clásica transmisión de conocimiento del profesor al alumno. En estos espacios se concibe el rol del docente como el de un facilitador, un tutor que guía y orienta al alumno para que este sea el constructor de su conocimiento, a través de instancias de trabajo individual y grupal.

A pesar del potencial de estos espacios es necesario tener presente que es posible encontrar -no pocas veces- modelos pedagógicos a distancia de primeras generaciones usando medios tecnologías de última generación (Bates, 2001; Moore, 2000; Baberà et.al. 2001). Es decir modelos lineales de transmisión de conocimiento en los cuales se usa los espacios virtuales para subir información "Hacer lo mismo de siempre usando tecnología punta es pobre a la vez que un gran error" (Duart y Martínez, 2001).

Las experiencias educativas que utilicen estos entornos virtuales de aprendizaje, tanto si se ubican en escenarios de enseñanza a distancia, presencial o mixta, implica una redefinición de los elementos organizativos del aprendizaje, en relación a: los agentes involucrados (profesores, participantes, administrativos); los espacios donde se lleva a cabo las actividades formativos (casa, centro educativo, aulas informáticas, lugar de trabajo); los tiempos; y secuencias de aprendizajes (Pérez, 2003). En relación al rol del tutor se plantean nuevas competencias y habilidades que estos profesionales deben dominar para utilizar el potencial pedagógico de estos espacios. Debe estar preparado para generar un dialogo efectivo con los participantes y entre los participantes, que favorezca el aprendizaje activo, la construcción de conocimiento cooperativo y/o colaborativo.

De acuerdo a Ryan, et.al. (2000) varios autores concuerdan en caracterizar los roles y responsabilidades del moderador en la CMC en cuatro categorias: pedagógica, social, administrativa y técnica. En lo pedagógica el tutor es un facilitador que contribuye con conocimiento especializado, focaliza la discusión en los puntos críticos, hacer las preguntas y responder a las contribuciones de los participantes, le da coherencia a la discusión, sintetiza los puntos destacando los temas emergentes. Salmon (2000) ha contribuido en gran medida a comprender el rol del moderador y sus cualidades y habilidades, estableciendo un modelo para la moderación de CMC que considera 5 etapas: acceso y motivación, socialización, compartir información, construcción de conocimiento y desorrollo. Estas etapas ilustran la interacción entre competencia y factores afectivos como crecimiento de la confidencia, motivación y dinámica de grupo (Macdonald, 2003)

Para Swan y otros colaboradores que investigaron los factores de éxito de los ambientes de aprendizajes online asincrónicos, hay tres factores que contribuyen significativamente, estos son: una interface transparente, un instructor que interactúe frecuentemente y constructivamente con los estudiantes, y una discusión valorada y dinámica (Swan y otros, 2000). El rol del tutor on-line o moderador de conferencia es crucial en el éxito de una aplicación de conferencia computacional para aprendizaje colaborativo. Para quiénes analizan las interacciones no tan solo desde el punto de vista cuantitativo, sino cualitativo, la frecuencia y calidad de las intervenciones en una CMC estará en gran medida marcada por las actividades moderadoras que efectúe el tutor (Perez, 2003).

\section{LA INVESTIGACIÓN: EI PROBLEMA Y LOS OBJETIVOS}

\subsection{El problema}


En Chile, de acuerdo a la información publicada por el Ministerio de Educación[2], en el sistema público 1.336, el 93\%, de los establecimientos de educación secundaria están conectados a la Red Enlaces[3], de ellos 1.157 el $87 \%$ cuentan con conexión Internet. Los profesores de estos establecimientos han sido capacitados en el uso de los recursos informáticos, entre ellos Internet. Por otra parte de acuerdo a un estudio reciente[4], un 64\% de los docentes, tiene equipamiento en su hogar y un $41 \%$ de ellos cuenta con conexión a Internet. Lo anterior llevó al Mineduc a plantearse la posibilidad de capacitar a docentes del sistema público por medio de cursos a distancia, haciendo uso de las tecnologías de la información y comunicación. Licitándose y ejecutándose el curso "funciones matemáticas en la enseñanza media"

El curso contempla un espacio virtual que contiene, materiales de apoyo, actividades y foro de discusión, además de herramientas de apoyo a la administración como la agenda y mensajería. En particular los ambientes de socialización han sido concebidos como espacios para enfatizar una reflexión compartida con el grupo acerca de los ejes temáticos del curso: reforma curricular, incorporación de las TIC, implementación de nuevos enfoques metodológicos, y evaluación de los aprendizajes. El texto inicial es generado por el equipo pedagógico siendo responsabilidad del tutor introducirlo al foro y animar su discusión. Uno de los aspectos deficitarios observados en la primera versión del curso -2002-, se relaciona con el bajo nivel de participación de los alumnos en los espacios destinados a la discusión y reflexión. En el 2003 se dictó una segunda versión en la cual se inserta este estudio.

\subsection{Objetivos}

Objetivo general: Conocer y caracterizar el rol pedagógico del tutor en la animación de los ambientes asíncronos de un espacio virtual de aprendizaje, en un curso de formación a distancia para profesores.

Objetivos Específicos:

- Conocer la evaluación de la actuación del tutor por parte de los alumnos del curso.

- Establecer categorías para identificar y analizar los tipos de intervenciones tutoriales realizadas por el tutor en el curso, en la animación de los diversos espacios virtuales asíncronos.

- Determinar cómo influye el rol del tutor en la decisión de los alumnos de abandonar o finalizar el curso.

- Determinar cuál es el tipo de uso queda el tutor a los diversos ambientes comunicativos asíncronos del curso.

\section{METODOLOGÍA}

Con el objeto de dar respuestas a los objetivos planteados en la investigación, se ha considerado como escenario de estudio el curso "Funciones matemática en la enseñanza media [5]. La experiencia que investigamos se enmarca en la segunda versión del curso, dictada el segundo semestre del 2003, del 14 de Junio al 5 de Diciembre, seleccionándose de allí 2 cursos. Los tutores era la segunda vez que participan en este rol en curso, los dos habían sido tutores del curso en la versión 2002. En relación a los alumnos la mayoría era primera vez que realizaban una experiencia de formación apoyado por TIC $85,7 \%$ (22), sólo un $14,3 \%$ (4) lo había hecho con anterioridad. Un 7,7\% (2) realizó el curso la vez anterior pero por diversos motivos no lo concluyó.

Al inicio del curso en la primera presencial a todos los alumnos se aplicó un primer cuestionario para obtener información acerca de su perfil profesional, conocimiento y uso de TIC, experiencias previas en formación a distancia a través de Internet, y sus expectativas frente al curso, analizando la información en forma conjunta. Durante el desarrollo del curso fueron encuestados los alumnos que abandonaron -8 a 10 semanas de iniciado el curso- los alumnos que finalizan -18 a 22 semanas-, con 
el objeto de evaluar el curso, sus componentes, el rol del tutor y las razones para abandonar o finalizar. Se analizó la información de ambos tipos de alumnos para los dos cursos en forma conjunta, las preguntas propias para los que finalizan se analizan en forma independiente.

Respecto a las intervenciones tutoriales, primero se realizó un análisis cuantitativo respecto al uso de los tres ambientes: foro, mensajería y agenda, para las 4 unidades del curso. En el caso del foro se cuantificó las intervenciones de los alumnos y tutores. En segundo lugar se establecieron categorías de análisis, tomando como cuerpo de análisis las intervenciones del tutor 1 , en el foro, la agenda y mensajería, correspondiente a la primera unidad del curso desarrollada las 9 primeras semanas, dado que esta es la que registra el mayor número de intervenciones. La categorización se realizó utilizando métodos deductivo e inductivo. En el primer caso se ha tomado el modelo de Salmon(2000), para lo segundo a partir de las intervenciones tutoriales observadas en el curso, se han modificado algunas de estas categorías, uniéndolas o subdividiéndolas, además se han introducido categorías adicionales como: retroalimentación, orientación e información. Las categorías se describen en la siguiente tabla.

Tabla 1: Categorías de Análisis Intervenciones Tutoriales

\begin{tabular}{|c|c|}
\hline Etapa & Categoría \\
\hline $\begin{array}{l}\text { Bienvenida } \\
\text { socialización } \\
\text { línea }\end{array}$ & $\begin{array}{l}\text { y Corresponde a los mensajes en los cuales se } \\
\text { nda una bienvenida al espacio virtual, se invita } \\
\text { a participar en él y se da una acogida de } \\
\text { alumno. Se busca conocer a los alumnos y que } \\
\text { ellos se conozcan entre si presentándose } \\
\text { (nombre, establecimiento, formación, años de } \\
\text { experiencia, motivaciones, expectativas frente } \\
\text { al curso, etc,) y que se sientan acogidos. }\end{array}$ \\
\hline Apoyo Técnico & $\begin{array}{l}\text { Ayuda en la solución de aspectos técnicos } \\
\text { relacionados con la configuración y acceso al } \\
\text { espacio virtual, además del uso de la } \\
\text { plataforma para envío de trabajo, publicación } \\
\text { de documentos, revisión de evaluaciones, etc. } \\
\text { Se ofrece ayuda (correo electrónico, teléfono, } \\
\text { horarios de contacto en plataforma). }\end{array}$ \\
\hline Motivación & $\begin{array}{l}\text { Intervenciones orientadas a incentivar el uso } \\
\text { de los espacios virtuales (foro, chat) aclarando } \\
\text { su rol cómo se relaciona con el resto del curso } \\
\text { y la cantidad y/o tipo de intervenciones } \\
\text { esperadas. Así mismo cómo acceder y usar } \\
\text { éstos espacios. Además contempla motivación } \\
\text { personal o grupal para el desarrollo de las } \\
\text { diferentes actividades del curso. }\end{array}$ \\
\hline $\begin{array}{l}\text { Intercambio } \\
\text { información }\end{array}$ & $\begin{array}{l}\text { Corresponden a las intervenciones orientadas } \\
\text { a incentivar la participación e intercambio de } \\
\text { información. }\end{array}$ \\
\hline $\begin{array}{l}\text { Construcción } \\
\text { conocimiento }\end{array}$ & $\begin{array}{l}\text { Intervenciones orientadas a presentar y animar } \\
\text { la discusión en línea como: presentar } \\
\text { elementos de discusión, reunir contribuciones } \\
\text { de los participaciones (para coleccionar } \\
\text { afirmaciones y relacionarlas con los } \\
\text { contenidos del curso), resumir las } \\
\text { contribuciones y relacionarlas con los } \\
\text { contenidos del curso,ampliar visiones y } \\
\text { proveer nuevos tópicos cuando se ha perdido } \\
\text { el rumbo, estimular nuevas ideas de discusión, }\end{array}$ \\
\hline
\end{tabular}




\begin{tabular}{|l|l|}
\hline \multirow{5}{*}{} & $\begin{array}{l}\text { introducir nuevos temas y sugieren } \\
\text { aproximaciones alternativas, ayudar a los } \\
\text { participantes en volverse los autores en lugar } \\
\text { de transmisores de conocimiento. }\end{array}$ \\
\hline $\begin{array}{l}\text { Evaluación crítica } \\
\text { del } \\
\text { (Desarrollo) }\end{array}$ & $\begin{array}{l}\text { Intervenciones orientadas a que los } \\
\text { participantes reflexionen sobre el aprendizaje } \\
\text { en red y evalúen el accionar del curso, en } \\
\text { cuanto a actividades desarrolladas, aspectos } \\
\text { positivos y por mejorar. Se trata de tener una } \\
\text { visión más crítica del curso. }\end{array}$ \\
\hline Retroalimentación & $\begin{array}{l}\text { Intervenciones que orientan el trabajo en el } \\
\text { curso especialmente el desarrollo de las } \\
\text { actividades y entrega de retroalimentaciones } \\
\text { respecto a los trabajos desarrollados y/o } \\
\text { intervenciones. }\end{array}$ \\
\hline Orientación & $\begin{array}{l}\text { Intervenciones orientadas a entregar } \\
\text { información respecto al desarrollo de las } \\
\text { actividades y trabajos calificados. }\end{array}$ \\
\hline Información & $\begin{array}{l}\text { Intervenciones que sólo entregan información } \\
\text { respecto a fecha y horarios de las charlas } \\
\text { interactivas o tiempo de duración del foro, } \\
\text { temas a tratar. Considera además información } \\
\text { de los aspectos administrativos del curso } \\
\text { como: fecha de entrega de trabajos, fechas de } \\
\text { presénciales, planificación de actividades, } \\
\text { recepción de trabajos, publicación de notas. }\end{array}$ \\
\hline
\end{tabular}

Con esta categorización se categorizó las intervenciones tutoriales en los tres ambientes: el foro, agenda y mensajerías, apoyándose por el software ATLAS-TI. Esto nos facilitó la cuantificación de la presencia de las diversas categorías en cada uno de los espacios y la obtención de ejemplos que nos permiten analizar cada categoría y ver como ellas se presentan en la acción tutorial. Las categorías al considerar como unidad de análisis cada intervención tutorial en su conjunto no son excluyentes, existiendo casos en que las intervenciones tutoriales responden a más de una categoría a la vez. En estos casos se optó por asignar las categorías correspondientes, no forzando la clasificación a categorías exclusivas de modo de recoger de la forma más fiel posible, la intencionalidad de las intervenciones tutoriales.

\section{ANÁLISIS DE LOS RESULTADOS}

\subsection{Alumnos participantes}

El curso se inicia con una sesión presencial, allí se aplicó el primer cuestionario, del total de cuestionario respondidos, se han considerado los de los alumnos activos, correspondientes a los que tienen contabilizados minutos de conexión. Ellos alcanzan a 14 para el curso 1 y 12 para el curso 2.

La mayor parte de los alumnos 61,54\%, corresponden al sexo masculino. Provienen de establecimientos de dependencia particular subvencionada un 57,7\%, un 38,5\% provienen de establecimientos municipales, y un 3,9\% de enseñanza superior.

En relación al uso de las TIC se consulto por 14 indicadores los que se evaluaron en una escala Liker relacionada al nivel de dominio del recurso específico (5=muy bien, $4=$ Bien, $3=$ Regular, $2=$ Mal, y $1=$ No lo domina). El dominio general de las TIC es de un 3,23, siendo mayor en el Curso 2 con un promedio de 3,38, en relación al Curso 1 que registro 3,07. A nivel general los recursos que se reportan con un mejor nivel de uso son el procesador de textos 4,04 y búsqueda de información y 
recursos en Internet 3,86, también destacan los recursos asociados a Internet como el correo electrónico y navegación en Internet con un promedio de 3,85 respectivamente. Los más bajos son: el uso de editores gráficos 2,14 y uso de comprimidores 2,36 .

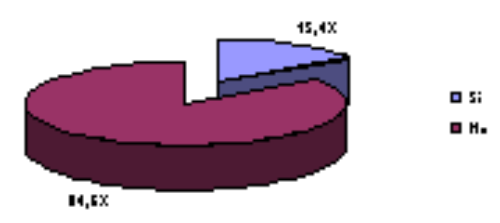

Gráfico 1: Experiencia en formación usando TIC
En relación a la experiencia en formación haciendo uso de las TIC. El $84,6 \%$ no registran experiencia, si la tiene el $15,4 \%$. Tendencia que es similar en cada curso. De los 4 alumnos con experiencia el $50 \%$ la consideran buena, un $25 \%$ muy buena y un $25 \%$ regular.

El lugar desde donde se accede al curso es un elemento clave en el éxito del mismo, el tener acceso a la tecnología es una condición básica para trabajar en el curso. Respecto al lugar de conexión, un $46,2 \%$ se conectarán desde sus hogares, un 26,9\% mencionan el establecimiento. Estas tendencias se mantienen en los respectivos cursos.

Las razones o motivaciones de los alumnos para participar en el curso, son un buen indicador de los intereses que los llevan a ingresar a él. La mayor parte manifiesta que se inscribió en el curso para experimentar una nueva forma de aprendizaje un 76,9\% la otra razón es la descripción del curso un 46,2\%. Las razones menos influyentes son la exigencias del establecimiento y haberlo dejado inconcluso la vez anterior, ambas representan un 7,7\%.

Respecto al grado de interés de los alumnos por los diversos aspectos contemplados en el curso, se presentaron 9 indicadores los cuales se evaluaron con una escala Liker (5=Mucho interés a $1=$ No hay interés) En general el interés es alto llega a un promedio de 4,63, siendo mayor en el Curso 1 con 4,92 en relación al Curso 2 que marco un promedio de 4,33.

\subsection{Alumnos que abandonan y finalizan}

Estos alumnos fueron encuestados en dos instancias diferentes, los que abandonaron al termino de la primera unidad, aproximadamente 8 a 10 semanas de iniciado el curso, los que finalizaron después del termino de la unidad 3 y previo al termino del curso aproximadamente de la semana 18 a la 22 . Se analizó la información de ambos tipos de alumnos para los dos cursos en forma conjunta dada que gran parte de las preguntas son las mismas. Esto nos permite contrastar la información proporcionada por estos grupos de alumnos que tomaron caminos distintos frente a la experiencia investigada en ambos cursos.

En ambos cursos se contó con alumnos en ambas categorías es decir: abandonaron y finalizaron. La tabla 2 muestra el total de alumnos de cada curso señalando los que abandonaron y finalizaron indicándose en cada caso la descomposición general de cada curso y los alumnos que respondieron los respectivos instrumentos.

Tabla 2: Alumnos participantes de la investigación

\begin{tabular}{|c|c|c|c|c|c|c|c|c|c|}
\hline Tipo de & \multicolumn{3}{|c|}{ Curso 1 } & \multicolumn{3}{c|}{ Curso 2 } & \multicolumn{3}{c|}{ Total Generales } \\
\cline { 2 - 10 } Alumnos & Frec. & Responden & $\%$ & Frec. & Responden & $\%$ & Frec. & Responden & $\%$ \\
\hline Abandonaron & 5 & 4 & $80,0 \%$ & 7 & 6 & $85,7 \%$ & 12 & 10 & $83,3 \%$ \\
\hline Finalizaron & 9 & 8 & $88,9 \%$ & 5 & 3 & $60,0 \%$ & 14 & 11 & $78,6 \%$ \\
\hline Total & 14 & 12 & $85,7 \%$ & 12 & 9 & $75,0 \%$ & 26 & 21 & $80,8 \%$ \\
\hline
\end{tabular}


a) Actitud hacia el curso

Tanto los alumnos que abandonan como los que finalizan conocieron el curso y utilizaron sus diversos recursos, claro esta que en diferentes medidas, dado el tiempo de permanencia en el mismo alrededor de 6 semanas para los que abandonan y 24 para los que finalizan. En general un 61,9\% manifiesta una actitud positiva hacia el curso, manifiestan una actitud negativa un 14,3\%. La actitud positiva alcanza a un $40 \%$ para los que abandonan y un $81,8 \%$, para los que finalizan.

b) Acción Tutorial

El actuar del tutor (tabla 3) se midió por medio de diversos indicadores que buscaron establecer cómo evalúan los alumnos el rol del tutor en: el apoyo en los aspectos técnicos, el apoyo en los contenidos, el apoyo en la construcción de conocimiento, la animación de la comunidad virtual de aprendizaje y la animación de los espacios de trabajo.

Tabla 3: evaluación de la acción tutorial

\section{Abandonan Finalizan}

\section{Curso 1 Curso 2 Total Curso 1 Curso 2 Total Totales}

MediaDesv.MediaDesv.MediaDesv.MediaDesv.MediaDesv.MediaDesv.MediaDesv.

1. El apoyo

para la

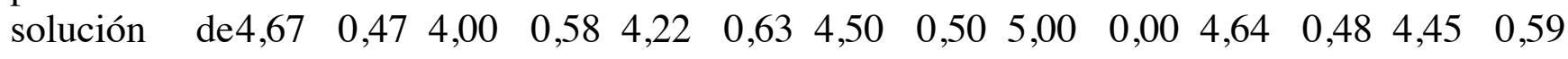
problemas

técnicos

2. El apoyo

$\begin{array}{lllllllllllllll}\text { en } & \operatorname{los}_{4,00} & 0,71 & 4,00 & 0,58 & 4,00 & 0,63 & 4,38 & 0,48 & 5,00 & 0,00 & 4,55 & 0,50 & 4,29 & 0,63\end{array}$ del curso

3. Capacidad

para crear $\mathrm{y}$

mantener un

ambiente de

$\begin{array}{lllllllllllllll}\text { trabajo } & \mathrm{al}_{4,00} & 0,71 & 4,00 & 0,82 & 4,00 & 0,77 & 4,75 & 0,43 & 5,00 & 0,00 & 4,82 & 0,39 & 4,43 & 0,73\end{array}$

una

comunidad

de

aprendizaje

4. La

animación de

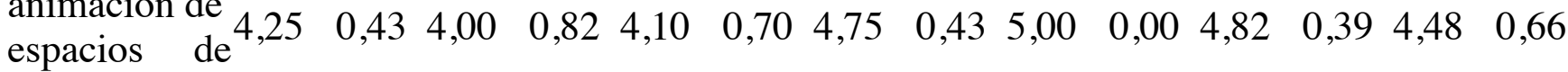

comunicación

5. El apoyo

para la

$\begin{array}{lllllllllllllll}\text { construcción } & 4,00 & 0,71 & 4,00 & 0,58 & 4,00 & 0,63 & 4,25 & 0,43 & 5,00 & 0,00 & 4,45 & 0,50 & 4,24 & 0,61\end{array}$

conocimiento

en el curso

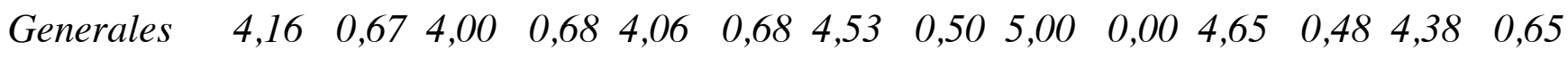

Escala Liker 5=Muy bueno, 4=Bueno, 3 =Regular, 2=Malo, 1=Muy malo

En términos generales hay una valoración positiva del actuar del tutor que se refleja en un promedio de 4,38. Siendo más elevado el promedio que asignan los alumnos que finalizan 4,65 en relación a los 
que abandonan 4,06. En ambos cursos se repite el fenómeno siendo significativo en el curso 2 donde se registra una diferencia de 1 punto entre los alumnos que abandonan y los que finalizan.

El aspecto mejor evaluado es la animación de los espacios de trabajo (4). El menor valorado el apoyo del tutor en la construcción de conocimiento (5) lo que de alguna forma es coherente con el escaso uso del foro para el intercambio de información. Por tipo de alumnos el mejor evaluado para los que abandonan es el aspecto apoyo técnico (1) y el menor evaluado los puntos (2), (3) y (5). Para los que finalizan los mejores evaluados son los puntos (3) y (4) relacionados con la animación del ambiente en la comunidad de aprendizaje y los espacios de comunicación y el peor evaluado el punto 5 relacionado al apoyo en la construcción de conocimiento.

c) Uso del foro

En términos generales el foro lo uso un $85,7 \%$ y no lo uso el $14,3 \%$. En relación a los curso el nivel de uso es más o menos similar un $83, \%$ en el curso 1 y un $88,9 \%$ en el curso 2 . El $70 \%$ de los que abandonan informan haberlo usado, en contraste con el $100 \%$ de los que finalizan.

En cuanto al uso del foro, en general lo uso sólo para lectura un 55,6\% y para lectura e intervención el 44,4\%. En relación a los curso el nivel es disímil, en efecto se usó para solo lectura en el curso 1 un $30 \%$ y en el curso 2 un 87,5\%, para lectura e intervención un $70 \%$ en el curso 1 y un $12,5 \%$ en el curso 2. Los que finalizan lo usan para lectura e intervención un 45,5\%, en tanto un 42,9\% de los que abandonan informan haberlo usado con esta finalidad.

Una de las utilidades del foro es apoyar al alumnos en la construcción de conocimiento. La grafica siguiente muestra la opinión de los alumnos que finalizan el curso respecto a la utilidad del foro en el proceso de construcción de conocimiento.

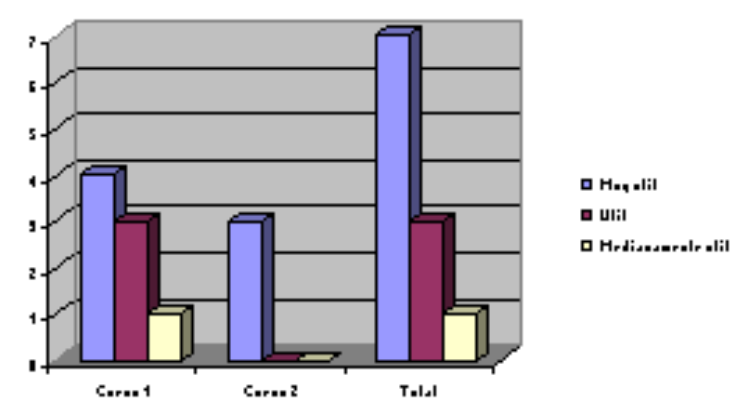

Grafico 2: Utilidad del foro en la construcción de conocimiento
La utilidad es evaluada positivamente por el $81,9 \%$, considerada muy útil por el $63,6 \%$ y útil por el $27,3 \%$. La opción neutra medianamente útil alcanza a $9,1 \%$.

En el curso 1, el 50\% lo considera muy útil y el y 37,5 útil, sumando esta apreciación positiva un $77,5 \%$. En el curso 2 el $100 \%$ lo considera muy útil.

d) El rol del tutor en la animación del foro

Se evaluó el grado de acuerdo o desacuerdo de los alumnos respecto a ciertas tareas consideradas relevantes en la animación del foro por parte del tutor. La siguiente tabla muestra los resultados obtenidos.

Tabla 4: evaluación del rol del tutor en la animación del foro por tipo de alumnos y cursos

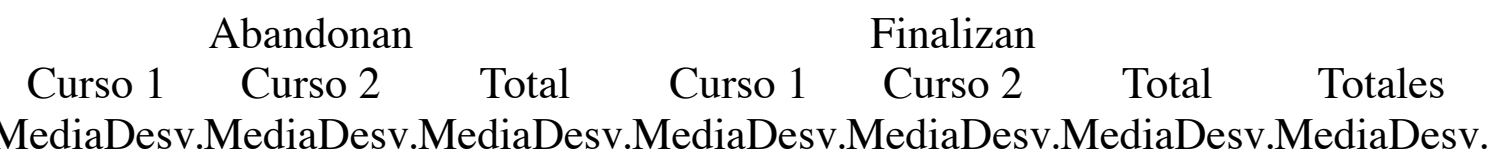

Introdujo

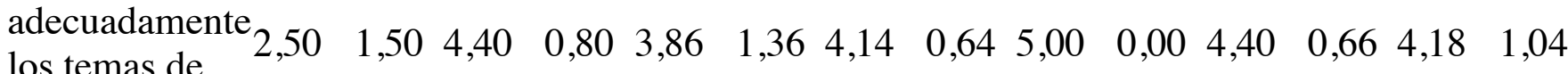

discusión 
Resumió los

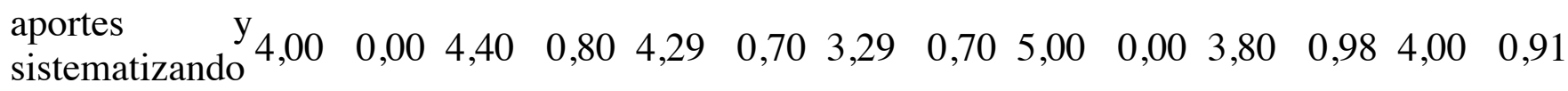
la información

Reorientó la

discusión de

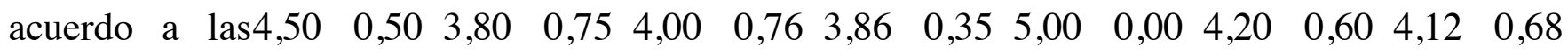
intervenciones

de los alumnos

Favoreció el

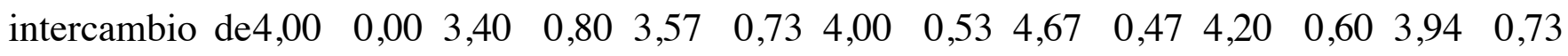

información

Ayudó a la

creación de

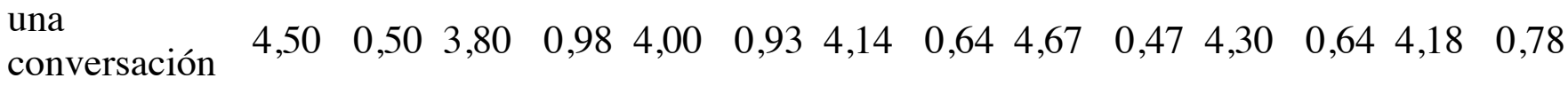

interesante $\mathrm{y}$

aportadora

Resultados

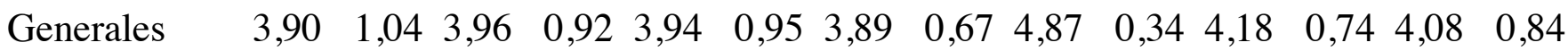

Escala Liker de 5 opciones $5=$ totalmente de acuerdo a $1=$ totalmente en desacuerdo

El promedio general alcanza a 4,08, siendo más positivo para los que finalizan 4,18 respecto a los que abandonan 3,94. Dentro de los que abandonan los promedios por cursos son parejos 3,90 y 3,96 respectivamente. Para los que finalizan las apreciaciones es disímil 3,89 en el curso1 y 4,87 en el curso2.

e) Ambiente de mensajería

Este ambiente de trabajo permitía la comunicación vía mensajes entre el tutor y los alumnos. Un alto $\%$ reconoce que recibieron información por parte del tutor por esta vía un $65 \%$ señala que siempre y un $25 \%$ casi siempre, sumando estas dos opciones el $90 \%$. Al observar los datos por cursos y tipos de alumnos se tiene una valoración positiva, que alcanza a $77,8 \%$ para los que abandonan y $100 \%$ para los que finalizan.

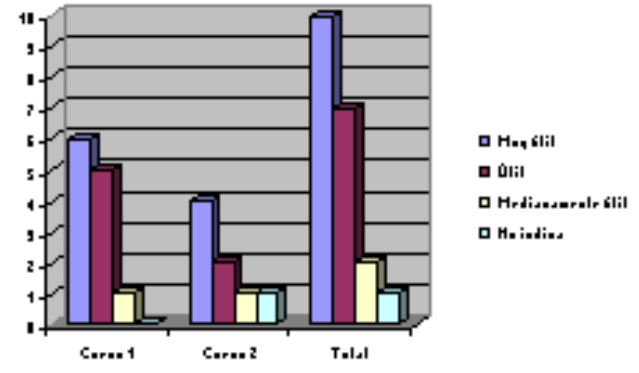

Grafico 3: Utilidad de la mensajería

f) Aspectos generales del Curso
Los apreciación respecto a la utilidad de la información que les hizó llegar el tutor por esta vía es en general evaluada positivamente por el $85 \%$, considerándola muy útil el $50 \%$ y útil el $35 \%$.

La utilidad es evaluada como positiva por el $77,7 \%$ de los que abandonan y por el $90,1 \%$ de que finalizan.

En relación a seguir un proceso de formación a distancia a través de Internet en temas matemáticos, los alumnos presentan una valoración positiva. En efecto el 47,6\% lo considera muy adecuado y el $23,8 \%$ adecuado lo que suma un $71,4 \%$ como apreciación positiva. De acuerdo a los alumnos que abandonan y finalizan la apreciación es positiva siendo superior para las segundos con un $81,8 \%$ frente al $60 \%$ de los primeros.En general la apreciación de la plataforma de formación del curso es 
positiva para el $81 \%$. Para los que abandonan la apreciación positiva en un $70 \%$ y un $90,9 \%$ para los que finalizan.

Para los alumnos que finalizan el curso se realizó dos preguntas relacionadas a la percepción de los alumnos respecto a la metodología de trabajo propuestas por el curso y el aprendizaje en forma colaborativa.

Respecto a la consulta en qué medida la metodología de trabajo lo situó al centro del aprendizaje, siendo ellos quienes define en forma autónoma su camino de aprendizaje de acuerdo a sus intereses y motivaciones, construyendo conocimiento a través de la interacción con: los materiales, el tutor y los compañeros. Este énfasis de la metodología, es considerado positiva por un $81,8 \%$, el restante $18,2 \%$ la considera regular. Por cursos es notable las diferencias, mientras el curso 2 considera que la metodología logra lo propuesto en un 33,3\%, en el curso 1 esta apreciación alcanza al 87,5\%\%

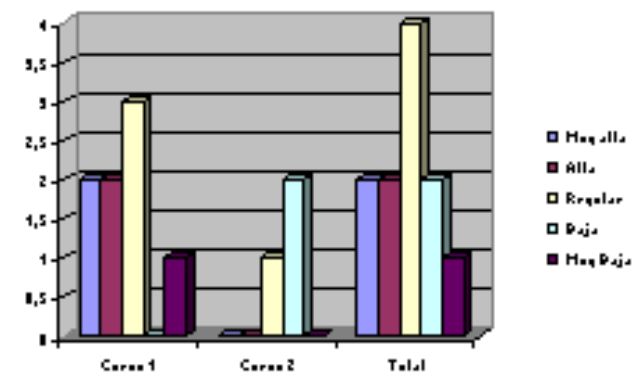

Grafico 4: Percepción logro aprendizaje colaborativo
En relación a la percepción del logro del aprendizaje en forma colaborativa, la mayor parte un $36,4 \%$ lo considera regular, el $27,3 \%$ baja o muy baja, y un $36,4 \%$ lo considera alta o muy alta. Por cursos es mejor la percepción el curso 1, logrando las opciones positivas muy alta y alta un acumulado de $50 \%$. En el curso 2 se centra en la opción baja con un $66,7 \%$.

g) Razones para el abandonar o finalizar el curso

La última parte de los instrumentos se destinó a recabar información sobre las razones que llevan a los alumnos a abandonarlo o finalizarlo, para esto se presentó afirmaciones relacionadas con 9 factores. Los que se evaluaron en una escala Liker de 3 opciones ( $1=$ No influye, $2=$ Influye, $3=$ Muy influyente). Para los alumnos que abandonan se obtienen los siguientes resultados.

Tabla 5: factores para la deserción por curso

Curso 1 Curso 2 Total

MediaDesv.MediaDesv. MediaDesv.

1. Problemas de conexión al curso (clave, conexión a

Internet, la pagina del curso no funciona, etc)

$\begin{array}{llllll}1,75 & 0,83 & 2,00 & 0,82 & 1,90 & 0,83\end{array}$

2. Bajo manejo de los contenidos del curso

$\begin{array}{llllll}1,50 & 0,50 & 1,50 & 0,76 & 1,50 & 0,67\end{array}$

3. Nivel de manejo de las competencias en el uso de Internet y los recursos tecnológicos en general

$\begin{array}{llllll}1,25 & 0,43 & 1,33 & 0,47 & 1,30 & 0,46\end{array}$

4. Falta de tiempo para conectarse al curso,

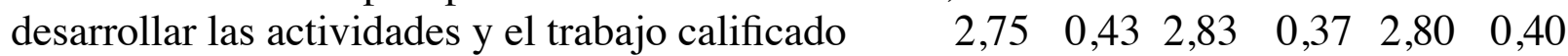

5. Horarios posibles de conexión inadecuados (muy tarde, los fines de semana, al final de la jornada escolar)

6. Falta de apoyo del establecimiento en el que trabaja para facilitar la conexión y tiempo de trabajo

7. El curso no satisfacía mis expectativas

8. Falta de apoyo y orientación del tutor para el desarrollo de las actividades y el trabajo calificado 
9. Las exigencias del curso en cuanto al trabajo que se evalúa son muy altas

$\begin{array}{llllll}1,00 & 0,00 & 1,67 & 0,47 & 1,40 & 0,49\end{array}$

Generales $\quad 1,72 \quad 0,83 \quad 1,78 \quad 0,81 \quad 1,76 \quad 0,83$

La influencia general de los factores señalados alcanza a un 1,76. Se observa que los factores señalados como más influyentes son los 4 y 5 relacionados con los el tiempo y los horarios de conexión Los menos influyente son el factor 8 relacionado al apoyo tutorial y el factor 3 vinculado al nivel de manejo de competencias tecnológicas. Para los alumnos que finalizan se obtienen los siguientes resultados.

Tabla 6: factores para la deserción por curso

Curso 1 Curso 2 Total

MediaDesv.MediaDesv.MediaDesv.

1. La superación o el no contar con problemas técnicos (clave de acceso, conexión a Internet, página del curso operativa, etc)

$\begin{array}{llllll}2,25 & 0,83 & 2,00 & 0,82 & 2,18 & 0,83\end{array}$

2. Su adecuado manejo de los contenidos considerados en el curso.

$\begin{array}{llllll}2,38 & 0,70 & 2,33 & 0,47 & 2,36 & 0,64\end{array}$

3. Un nivel adecuado de manejo de las competencias en el uso de Internet y los recursos tecnológicos en general.

4. Disponer o generarse el tiempo para conectarse al curso, desarrollar las actividades y el trabajo calificado.

$$
\begin{array}{llllll}
2,38 & 0,70 & 2,33 & 0,47 & 2,36 & 0,64
\end{array}
$$

5. La posibilidad de construir conocimiento a partir del trabajo colaborativo en el trabajo con el grupo curso o grupos pequeños.

$$
\begin{array}{llllll}
2,13 & 0,78 & 1,67 & 0,47 & 2,00 & 0,74
\end{array}
$$

6. Apoyo del establecimiento en el que trabaja para $\begin{array}{llllll}2,38 & 0,70 & 2,67 & 0,47 & 2,45 & 0,66\end{array}$ facilitar la conexión y tiempo de trabajo.

7. El curso cumple mis expectativas.

$\begin{array}{llllll}1,75 & 0,83 & 1,67 & 0,94 & 1,73 & 0,86\end{array}$

$\begin{array}{llllll}2,25 & 0,83 & 2,33 & 0,47 & 2,27 & 0,75\end{array}$

8. El apoyo y orientación del tutor para el desarrollo de las actividades y el trabajo calificado.

9. Los trabajos calificados son un aporte a su labor pedagógica y coherentes con la propuesta metodológica del curso.

$$
\begin{array}{lllllll}
\text { General } & 2,24 & 0,77 & 2,26 & 0,70 & 2,24 & 0,75
\end{array}
$$

El promedio general de influencia alcanza a un 2,24. Los factores más influyentes son el 4 relacionado con el tiempo para el desarrollo del curso y el 9 relacionado con los trabajaos calificados. En el curso 2 aparecen los mismos factores agregándose el apoyo del tutor, en el curso 1 se adicionan los factores relacionados con el manejo de las TIC y contenidos.

Los menos influyentes el factor 6 apoyo del establecimiento y el 5 relacionado a la posibilidad de construir conocimiento en forma colaborativa.

Se les pidió a los alumnos que finalizaron indicaran tres aspectos positivos del curso, los dos más mencionados: la metodología contenidos y actividades $24 \%$, el apoyo del tutor $16 \%$. De la misma forma se les pidió tres aspectos a mejorar, los dos más mencionados: fechas de realización del curso y entrega de trabajos $27 \%$, intercambio y trabajo colaborativo $18 \%$.

\subsection{El uso de los ambientes del curso}

Esta componente describe el uso de foros, agenda y mensajería. En el primero se recoge las intervenciones de tutores y alumnos, para los otros dos, sólo las tutoriales. 
El foro es el espacio privilegiado para el desarrollo la discusión, intercambio de información y construcción de conocimiento colaborativo. La tabla 7 muestra las intervenciones en los 4 foros, para el primero se tomó en cuenta el total de alumnos participantes es decir 26, 14 en el curso 1 y 12 en el curso 2. A partir del foro 2 la información se refiere a los alumnos activos, esto es, 9 en el curso1 y 5 en el curso 2. En general las aportaciones de los alumnos, alcanzando a un $57,1 \%$ y las de los tutores el $42,9 \%$.

Tabla 7: Mensajes en los foros

\begin{tabular}{|c|c|c|c|c|c|c|}
\hline \multirow[t]{2}{*}{ Foros } & \multirow[t]{2}{*}{ Curso } & \multirow{2}{*}{$\begin{array}{c}\mathrm{N}^{\mathrm{o}} \text { total de } \\
\text { Intervenciones }\end{array}$} & \multicolumn{2}{|c|}{ Alumnos } & \multicolumn{2}{|l|}{ Tutor } \\
\hline & & & $\begin{array}{c}\mathrm{N}^{\mathrm{o}} \mathrm{de} \\
\text { Aportaciones }\end{array}$ & $\%$ & $\begin{array}{c}\mathrm{N}^{\mathrm{o}} \mathrm{de} \\
\text { Aportaciones }\end{array}$ & $\%$ \\
\hline \multirow[t]{3}{*}{ Uno } & 1 & 29 & 16 & $55,2 \%$ & 13 & $44,9 \%$ \\
\hline & 2 & 16 & 7 & $43,8 \%$ & 9 & $56,2 \%$ \\
\hline & Totales & 45 & 24 & $53,3 \%$ & 21 & $46,7 \%$ \\
\hline \multirow[t]{3}{*}{ Dos } & 1 & 9 & 5 & $55,6 \%$ & 4 & $44,4 \%$ \\
\hline & 2 & 13 & 10 & $76,9 \%$ & 3 & $23,1 \%$ \\
\hline & Totales & 22 & 15 & $68,2 \%$ & 7 & $31,8 \%$ \\
\hline \multirow[t]{3}{*}{ Tres } & 1 & 11 & 8 & $72,7 \%$ & 3 & $27,3 \%$ \\
\hline & 2 & 5 & 3 & $60,0 \%$ & 2 & $40,0 \%$ \\
\hline & Totales & 16 & 11 & $68,8 \%$ & 5 & $31,3 \%$ \\
\hline \multirow[t]{3}{*}{ Cuatro } & 1 & 9 & 5 & $55,6 \%$ & 4 & $44,4 \%$ \\
\hline & 2 & 12 & 7 & $58,3 \%$ & 5 & $41,7 \%$ \\
\hline & Totales & 21 & 12 & $57,1 \%$ & 9 & $42,9 \%$ \\
\hline
\end{tabular}

En el foro de la unidad 1, se observa una mayor participación del curso 1, alcanzando a un 55,2\% del total de intervenciones, en el curso 2 son más las intervenciones tutoriales alcanzando un 56,2\%. El foro de la unidad 2, registra intervenciones disímiles en ambos cursos, duplicando el curso 2 las aportaciones del curso 1, observándose respecto al foro 1, un drástico descenso, aproximadamente un $48,9 \%$, esto puede deberse a que a estas alturas ya los cursos contaban con las respectivas deserciones. Las intervenciones tutoriales disminuyen un 66,6\% y la de los alumnos se reduce en $37,5 \%$.

El foro de la unidad 3 mantiene un comportamiento similar al observadas en el foro 2, aunque el número de intervenciones a nivel general y para ambos actores se reduce, en un $27 \%$, los tutores un $26 \%$ y los alumnos un 29\%. El foro de la unidad 4 presenta tendencias al alza, en las intervenciones totales subiendo en general un $31,5 \%$, la de los tutores un $80 \%$ y la de los alumnos un $9 \%$.

\section{b) Agenda}

La agenda es una herramienta que permite mantener informado a los alumnos periódicamente de los aspectos más relevantes en el desarrollo del curso. En la tabla 8 se muestra la información relativa al número de intervenciones en la agenda por parte de los tutores de ambos cursos.

Tabla 8: Intervenciones de los tutores en la agenda

\begin{tabular}{|l|c|c|c|c|c|c|}
\hline \multicolumn{2}{|c|}{} & \multicolumn{2}{|c|}{ Grupo 1 } & \multicolumn{2}{c|}{ Grupo 2 } & \\
\hline Unidades & Semanas & $\mathrm{N}^{\mathrm{o}}$ de & $\begin{array}{c}\text { Promedio } \\
\text { Semanal } \\
\text { Publicaciones }\end{array}$ & $\begin{array}{c}\mathrm{N}^{\mathrm{o}} \text { de } \\
\text { Publicaciones }\end{array}$ & Promedio & $\begin{array}{c}\text { Total de } \\
\text { Publicaciones }\end{array}$ \\
\hline Unidad 1 & 9 & 14 & 1,5 & 9 & 1 & 23 \\
\hline Unidad 2 & 7 & 7 & 1 & 7 & 1 & 14 \\
\hline
\end{tabular}




\begin{tabular}{|c|c|c|c|c|c|c|} 
Unıdad 5 & $\supset$ & 0 & 1,2 & 3 & 1 & 11 \\
\hline Unidad 4 & 6 & 6 & 1 & 7 & 1,2 & 13 \\
\hline General & 27 & 33 & 1,2 & 28 & 1,0 & 61 \\
\hline
\end{tabular}

Este espacio, se uso en promedio para enviar 1,1 mensajes por semana, siendo levemente más utilizado en el curso 1. Se observa en la primera unidad, una mayor frecuencia de intervenciones del Tutor1, en relación al Tutor2. Si bien ambos tutores entregan la misma información, en el primer caso se segmenta en mensajes más cortos y precisos. En la segunda Unidad se estandariza alrededor de 1 intervención semanal, siendo más escuetas las registradas por el tutor 1 y más extensas las del tutor 2.

c) Mensajes

La plataforma posee un ambiente para la comunicación vía mensajes llamada mensajería. Se la utiliza en forma similar al correo electrónico. A través de este espacio el tutor hace llegar mensajes a los alumnos, en forma grupal o individual. La siguiente tabla muestra el nivel de mensajes en ambos cursos.

Tabla 9: Intervenciones de los tutores en la mensajeria

Curso 1

Grupales Individuales Total

\section{Curso 2}

Grupales Individuales Total

\begin{tabular}{cccccccccccccc} 
Unidades & Cant. $\%$ & Cant & $\%$ & Cant & \multicolumn{4}{c}{ Cant $\%$} & Cant & $\%$ & Cant. & $\%$ \\
Unidad 1 & 19 & $27,5 \%$ & 50 & $72,5 \%$ & 69 & $66,3 \%$ & 11 & $30,6 \%$ & 25 & $69,4 \%$ & 36 & $41,4 \%$ \\
Unidad 2 & 7 & $38,9 \%$ & 11 & $61,1 \%$ & 18 & $17,3 \%$ & 12 & $57,1 \%$ & 9 & $42,9 \%$ & 21 & $24,1 \%$ \\
Unidad 3 & 3 & $30,0 \%$ & 7 & $70,0 \%$ & 10 & $9,6 \%$ & 4 & $44,4 \%$ & 5 & $55,6 \%$ & 9 & $10,3 \%$ \\
Unidad 4 & 2 & $28,6 \%$ & 5 & $71,4 \%$ & 7 & $6,7 \%$ & 5 & $23,8 \%$ & 16 & $76,2 \%$ & 21 & $24,1 \%$ \\
Tot. & 31 & $29,8 \%$ & 73 & $70,2 \%$ & 104 & $100,0 \%$ & 32 & $36,8 \%$ & 55 & $63,2 \%$ & 87 & $100,0 \%$
\end{tabular}

General

Se observa un mayor uso de los mensajes individuales respecto a los grupales en ambos cursos. En relación a los mensajes por unidad estos se distribuyen en forma diferente en cada curso, mientras que para el primer curso la unidad 1 concentra el 66,3\% y luego desciende drásticamente hasta llegar a la unidad 4 con un 6,7\%, en el segundo curso la unidad 1 concentra el 41, 4\% de los mensajes y luego se mantiene en $24,1 \%$ en las unidades 2 y 4 bajando sólo en la unidad 3 a un 10,3\%.

\subsection{Categorización intervenciones tutoriales}

Se analizó las intervenciones tutoriales para comprender el tipo de intervenciones que realiza el tutor, en los espacios de comunicación del curso. Con esta finalidad, se tomó como cuerpo de análisis las intervenciones del tutor1 en la Unidad 1, transformado las intervenciones en cada espacio -foro, agenda y mensajería- en un documento independiente. Sobre la base de las categorías definidas anteriormente en la metodología (Tabla 1), se procedió a categorizar los tres documentos, apoyándose por ATLAS-TI. Obteniendo una cuantificación de la presencia de las categorías en cada uno de los espacios y ejemplos de ellas. Los espacios de trabajo son utilizados por el tutor con diferentes énfasis como se muestra en la tabla 10, en ella se cuantifica las intervenciones tutoriales de acuerdo a las categorías de análisis para cada uno de los espacios.

Tabla 10: Intervenciones tutoriales según categorías de análisis

\begin{tabular}{|c|c|c|c|c|c|}
\hline Códigos & Agenda & Foro & Mensajería & Totales & $\%$ \\
\hline Apoyo Técnico & 1 & 0 & 5 & 6 & $6, \%$ \\
\hline Bienvenida y socialización & 0 & 0 & 10 & 10 & $11 \%$ \\
\hline $\begin{array}{c}\text { Construcción de } \\
\text { conocimiento }\end{array}$ & 0 & 10 & 0 & 10 & $11 \%$ \\
\hline Fvalıación crítica & 0 & 1 & 0 & 1 & $1 \%$ \\
\hline
\end{tabular}




\begin{tabular}{|c|c|c|c|c|c|} 
& $\sim$ & $*$ & $\sim$ & & \\
\hline Intercambio de información & 0 & 2 & 3 & 5 & $5 \%$ \\
\hline Motivación & 4 & 0 & 9 & 13 & $14 \%$ \\
\hline Orientación & 9 & 0 & 7 & 16 & $17 \%$ \\
\hline Retroalimentación & 0 & 0 & 14 & 14 & $15 \%$ \\
\hline Totales & 16 & 13 & 64 & 93 & $100 \%$ \\
\hline
\end{tabular}

En total se producen 93 intervenciones, de las cuales un $18 \%$ corresponden a la agenda, un $14 \%$ al foro y un $68 \%$ a mensajería. La mayor parte de las intervenciones tutoriales corresponden a las categorías información $(20 \%)$ y orientación $(17 \%)$. Las menos usadas son intercambio de información $(5 \%)$, apoyo técnico $(6 \%)$ y evaluación crítica (1\%). Para comprender las categorías de análisis de las intervenciones tutoriales, expondremos ejemplos de ellas indicando los espacios donde estas se presentan.

\section{a) Apoyo Técnico}

Esta categorías se presenta en el $6 \%$ del total de las intervenciones. Se presenta en dos de los ambiente, la agenda en la cual alcanza a un $6 \%$ y en la mensajería donde registra un $8 \%$. En el ejemplo extraído de la mensajería, se da apoyo entregando información al alumno para conseguir apoyo del tutor o del equipo técnico.

A:

TODOS

Enviado:

2003-06-26

Estimado Profesor y Colega: Espero que estés muy bien y que este día haya sido alentador y positivo tanto en lo personal como en lo laboral. Te envío los teléfonos que serán de utilidad durante el desarrollo del curso. Soporte técnico:

Mensaje: (02) 2400530 Consultora: (02) 3354455 Celular personal : 09 - 3303242 Soporte técnico debes comunicarte con Mabel Alarcón cuando tengas problemas técnicos con la Plataforma del curso. En la Consultora me ubicas los días Lunes, Martes y Jueves desde las 19:00 hasta las 22:00 hrs, En mi celular cuando necesites hacerlo, hazlo con toda confianza.

b) Bienvenida y Socialización en Línea

Esta categoría se presenta en un $11 \%$ del global de intervenciones y esta presente sólo en el ambiente de mensajería donde representa un $16 \%$. Existen dos formas diferentes de presencia de esta categoría una bienvenida genérica para todo el grupo curso y un mensaje generado una vez que el alumno responde al mensaje de bienvenida y envía la información solicitada. Se presenta un ejemplo del primer caso.

A:

Enviado:

Mensaje:

\section{c) Motivación}

Esta categoría ocupa el 14\% de las intervenciones. Se manifiesta en la agenda en cuyo espacio alcanza el $25 \%$ y en la mensajería donde ocupa el $14 \%$. El ejemplo esta tomado de la agenda, se motiva a los alumnos a participar en el foro y el espacio de publicaciones. 
Estimados Profesores y Colegas:

(..) No olvides participar del Foro de la Unidad 1 que cada vez se vuelve más interesante. Además de revisar las Publicaciones hechas por Profesores que han querido compartir su trabajo y que de alguna manera les ayudará para planificar el de ustedes.

Ánimo y Buena suerte

d) Intercambio de información

Esta categoría ocupa un 5\% del total de las intervenciones, presentándose en el foro, en el cual registra el $15 \%$ y en la mensajería en la cual alcanza el $5 \%$. El ejemplo extraído desde el foro, hace una invitación a buscar recursos en la Web y compartirlos en este espacio.

Autor: $\quad \begin{aligned} & \text { Tutora } \\ & \quad<\text { diva_pv@hotmail.com> } \quad \text { Fecha: 2003-07-13 }\end{aligned}$

Título: $\quad$ SITIOS WEB

Contenido:Es importante apoyar nuestra metodología de enseñanza de las funciones con diferente material novedoso que podemos encontrar navegando por Internet.

Les invito a compartir el material encontrado.

e) Construcción de conocimiento

Forman parte de esta categoría las intervenciones destinada a moderar la discusión introducir nuevas preguntas destinadas a reorientar la discusión y resumir los aportes. Esta categoría alcanza un 11\% del total de intervenciones, presentándose exclusivamente en el foro, en el cual ocupa el 77\%. En el ejemplo, se realiza una síntesis de la discusión recogiendo las opiniones vertidas por los participantes.

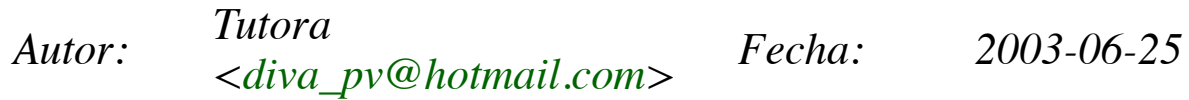

Título: metodología innovadora

Contenido:De acuerdo a todos las opiniones vertidas por ustedes podríamos concluir que el estudio de las funciones se inicia dando a los alumnos diferentes ejemplos de la vida diaria de manera de contextualizar el concepto para luego definirlo desde el punto de vista matemático. Sería importante que después de que los alumnos conocen la definición fuesen capaz de crear ejemplos?, Que concluiríamos con ello?, Es importante darle ejemplos que no son función?

El concepto de función lo define el profesor ?, El concepto de función lo construyen los alumnos después de analizar cada uno de los ejemplos dados?

f) Evaluación crítica del Curso

Esta categoría a nivel global ocupa un $1 \%$ de las intervenciones, presentándose sólo en el foro donde registra el $1 \%$ de las intervenciones. A pesar de no ser significativa, hemos querido rescatarla, porque apunta al nivel más alto de los niveles de la moderación en línea propuesta por Salmon (2000) correspondiente a la categoría de desarrollo. En ella los alumnos realizan una valoración crítica del ambiente de trabajo y la experiencia de formación en línea, si bien considera otros aspectos no presentados en esta unidad del curso como: reflexionar sobre el aprendizaje en red; relacionar la conferencia con acciones educativas posteriores. Se presenta este ejemplo donde se invita a los alumnos a evaluar esta primera unidad, rescatando fortalezas y debilidades, además se pide sugerencias para mejorar el curso. 
Autor: <diva_pv@hotmail.com> Fecha: 2003-08-08

Título: $\quad$ Análisis Cualitativo sobre la Unidad 1

Contenido:Los invito a formular algún comentario u opinión sobre la Unidad 1, en relación a las actividades sugeridas, documentos de apoyo de la unidad, fortalezas y debilidades en general. Al mismo tiempo solicito a cada uno de ustedes cualquier tipo de sugerencia que vaya en beneficio de esta propuesta o diseño de "Educación a Distancia".

g) Retroalimentación

Esta categoría a nivel global ocupa el $15 \%$ de las intervenciones, presentándose sólo en el ambiente de mensajería, en el cual alcanza el 22\%. El ejemplo muestra una retroalimentación correspondiente a la evaluación del trabajo calificado 1. En ella primero se indican los aspectos positivos y menos logrados del trabajo, y luego la evaluación global a partir de la ponderación fijada para el trabajo escrito y participación en los espacios de comunicación.

A:

Enviado:

Mensaje:

h) Orientación

Esta categoría registra un $17 \%$ del total de las intervenciones, se presenta en la agenda donde registra el $56 \%$ y en mensajería donde alcanza al $22 \%$. El ejemplo tomado de la agenda orienta acerca de las actividad que el alumno debería estar desarrollando durante la semana y los recursos de apoyo.

\section{Actividad 2}

Estimados Profesores y Colegas:

Les recuerdo que esta semana deben trabajar en el desarrollo de la actividad 2 "Contextualizar las funciones en el marco del nuevo enfoque de la enseñanza de la matemática escolar".

No olviden apoyarse con el material que hay en Biblioteca, participar de los recursos de la plataforma y visitar sitios web relacionados con la actividad.

\section{Buena suerte}

i) Información

Esta categoría a nivel general, registra un $20 \%$ de las intervenciones, se presenta en la agenda y mensajería alcanzando al $13 \%$ y $24 \%$, respectivamente de las intervenciones. El ejemplo tomado de la agenda informa acerca de los elementos generales publicados en el curso para guiar el trabajo en la unidad, como lo es, la "hoja de ruta".

\section{PUBLICACIÓN HOJA DE RUTA}

Estimados Profesores y colegas:

Deseo comunicarles que he publicado la Hoja de Ruta que los ayudará a planificar el tiempo para el desarrollo de las diferentes actividades de cada Unidad del curso. 
Esta Hoja de Ruta está publicada en el árbol de contenidos en la Unidad 1. Los invito a conocerla y leerla para poder cumplir los plazos propuestos.

\section{CONCLUSIONES}

La realización de experiencias de formación a distancia usando las TIC comienza a tomar fuerza, como una posibilidad valida para apoyar la formación continua de docentes. En este trabajo hemos analizado una de estas experiencias, focalizando en el rol del tutor en la animación de estos espacios virtuales. A continuación presentaremos algunas de las principales conclusiones obtenidas.

El rol del tutor no tiene mayor incidencia en la decisión de abandonar el curso, es el aspecto menos influyente para los alumnos que abandonan, pero si influye en la permanencia, siendo citado en tercer lugar por los que finalizan. En relación a las razones para abandonar las más influyentes son el: acceso a los recursos tecnológicos para comunicarse al curso y tiempo para el desarrollo de las actividades. Estas razones son coherentes con las presentadas por Leggett y Persichitte en relación con las barreras en la inserción de la tecnología en el aula de K-12, pues estos citan: tiempo, acceso, recursos y especialización (Leggett y Persichitte 1998 citado en Muilenburg y Berge, 2001). El factor tiempo es crucial, porque los alumnos que finalizan reconocen como uno de los elementos más influyentes en su decisión, el contar con el tiempo para el desarrollo de las actividades. Luego creemos que uno de los elementos que estas experiencias deben asegurar para optimizar su éxito, es el acceso real de los profesores a la tecnología y contar con los tiempos necesarios para desarrollar las actividades formativas.

La evaluación de la acción tutorial es positiva en su conjunto, siendo mejor evaluada por los alumnos que finalizan en relación a los que abandonan. Los roles del tutor en la animación de los diversos espacios en el ambiente de trabajo, el apoyo técnico y pedagógico, así como su capacidad para mantener y animar la comunidad de aprendizaje son aspectos valorados. Estos aspectos facilitan el mantener a los alumnos activos en el curso. Sin embargo desde la perspectiva pedagógica orientada a la construcción de conocimiento en forma colaborativa en red, el apoyo del tutor en la construcción del conocimiento es el aspecto menor evaluado. Esto es concordante con la escasa participación del tutor y los alumnos en los foros.

A pesar que el curso promueve el aprendizaje colaborativo, el logro de éste, es evaluado negativamente por los alumnos, siendo menor evaluado por los que abandonan. Esto es concordante con el escaso nivel de participación que registran los alumnos en el foro y la valoración negativa que se registra en términos de la facilidad para desarrollar aportes a la discusión del foro. Este aspecto tiene algunas explicaciones que podemos encontrar en la literatura, en términos, a que se necesita tiempo y práctica para adquirir las habilidades para desarrollar un trabajo colaborativo en red, además el uso de los espacios de intercambio depende de la interface, el rol del tutor en su animación y el valor de las discusión (Swan, et.al, 2000; Tolme y Boyle, 2000). Estos resultados no concuerdan con investigaciones en esta área, las que señalan que este tipo de experiencias en red, promueven la participación activa de los alumnos, las aportaciones al debate son reflexionadas, donde los alumnos elaboran ideas que presentan y discuten con los otros alumnos (Harasim et.al 2000; Crook, 1998)

$\mathrm{Al}$ analizar las intervenciones tutoriales podemos recurrir a modelos propuestos en la literatura como el de Salmon(2000), pero para responder a una experiencia concreta deben complementarse con otras, producto del análisis de las intervenciones producidas. Las categorías generadas permitieron categorizar las intervenciones tutoriales y así determinar cual es el objetivo de ellas, además de cuantificar su presencia como una forma de establecer los nivele de uso. Las categorías generadas son un primer paso, habría que utilizarlas en otras experiencias de formación docente en línea para intentar validarlas y generalizarlas.

El espacio de trabajo virtual de este curso ofreció tres canales de comunicación asíncrona. Al categorizar las intervenciones tutoriales se observa usos con propósitos diferentes de cada uno de llos, 
aspecto que se manifiesta en la cantidad de intervenciones y al tipo de intervención. El foro se utiliza principalmente para la construcción de conocimiento, las agenda para la orientación y mensajería para la retroalimentación. No sabemos hasta que punto los alumnos perciben estos énfasis y si estos serán los adecuados.

En términos generales tanto los alumnos que abandonan como los que finalizan tienen una valoración positiva del curso, siendo mayor para estos últimos. A pesar de las dificultades detectados, los niveles de deserción inicial, y a que no necesariamente se logra implementar un modelo pedagógico que promueva la construcción de conocimiento en red, los docentes han valorado y validado estas formas de formación. Seguramente en una segunda oportunidad que estos docentes participen de experiencias de este tipo estarán en mejores condiciones para afrontarlas, así como para integrarlas a sus propias prácticas.

Esta investigación, a nuestro juicio, posee ciertas limitaciones. La mayor parte de los estudios teóricos como las investigaciones directamente relacionadas con el rol del tutor en los entornos virtuales de aprendizaje son escasas, están relacionadas con ámbitos de educación formal y donde los alumnos son estudiantes del nivel superior de pre y postgrado. Los modelos de análisis propuestos obedecen a estos últimos contextos. Esto nos llevo a generar nuestra propia metodología e instrumentos para la recogida de datos cuantitativos y cualitativos. En el caso cuantitativo se validaron con expertos, para el caso cualitativo las categorías de análisis, aunque fueron elaboradas tomando como base los aporte teóricas y los sistemas de clasificación existentes, es una propuestas nueva, que requiere ser validada, aplicándose a futuras experiencias que permitan además perfeccionarla. A pesar de las limitantes metodológicas que pueda tener esta investigación, sería interesante, probar la metodología planteada en nuevos estudios. Estas futuras investigaciones deberían estar en el ámbito de la formación continua de docentes, analizar un mayor número de intervenciones -por ejemplo las de todo el curso-, deseablemente aplicable a diferentes casos, e incorporar al análisis otros aspectos de la acción tutorial que esta investigación no ha considerado, como por ejemplo la preparación previa de los tutores para gestionar un curso a distancia y el grado de conocimiento de los contenidos del curso.

Este trabajo se enmarcó dentro de una investigación descriptiva exploratoria que permite generar las bases para afrontar una investigación mayor, ampliándola tiempo de ejecución y cantidad de información analizada, comprobar si las categorías establecidas son factibles de generalizar en procesos formativos a distancia destinados a docentes. Se abren nuevas preguntas de investigación cómo ¿cuál será el comportamiento de profesores de otras áreas?, ¿se corroborarían los resultados obtenidos?, ¿qué resultados se obtendrían con profesores de otras culturas, tanto latinas como europeas?

\section{BIBLIOGRAFÍA}

- BARBERÀ, E. (Coord), BADIA, A y MOMINO, J.M. (2001). La incógnita de la educación a distancia, Barcelona, ICE Universidad de Barcelona/Horsori

- BATES, A.W. (1995). Technology open learning and distance education, London/NewYork, Routledge.

- CIDE-UAH Centro de Investigación y Desarrollo de la Educación-Universidad Alberto Hurtado (2003). Informe Final del Estudio Seguimiento de curso a distancia con nuevas tecnologías Comprendiendo la Naturaleza. Disponible en: http://www.redenlaces.cl/paginas/doc/EvalCurs.doc [2003, Julio20]

- CABERO, J. (2001). La aplicación de las TIC: ¿esnobismo o necesidad educativa?, Red Digital, 1. http://reddigital.cnice.mecd.es/1/firmas/firmas_cabero_ind.html

- CROOK, Ch. (1998). Ordenadores y aprendizaje colaborativo, Madrid, Morata/MEC Ministerio de educación y cultura (Título original: Computer and the collaborative experience of learning, London: 
Routledge, 1994)

- COOK, J. (2002). The Role of Dialogue in Computer-Based Learning and Observing Learning: An Evolutionary Approach to Theory. Journal of Interactive Media in Education (JIME) [En línea], 5. Disponible en: http://www-jime.open.ac.uk/2002/5/cook-02-5-paper.html [2003, Octubre 28]

- CROWE, D. y ZAND, H. (2000). Computers and undergraduate mathemaTIC 3: Internet resources, Computer y Education, 35 (2), 123-147.

- DUART, J. y MARTÍNEZ, M. (2001). Evaluación de la calidad docente en entornos virtuales de aprendizaje. Disponible en: http://www.uoc.edu/web/esp/art/uoc/0109041/duartmartin.html [2003, Abril 8]

- GIL FLORES, J. (1994). Análisis de datos cualitativos: Aplicaciones a la investigación educativa. Barcelona, PPU

- GOETZ, J,P. y LECOMPTE M. D. (1988). Etnografía y diseño cualitativo en investigación educativa, Madrid, Morata.

- GROS, B. (2002). Constructivismo y diseños de entornos virtuales de aprendizaje, Revista de Educación, 328, 225-247.

- HARASIM, L., HILTZ, S., TUROFF, M. y TELES, L. (2000). Redes de aprendizaje: Guía para la enseñanza y el aprendizaje en red, Barcelona, Gedisa/EDIUOC [Versión original: Learning networks. A fiel guide to teaching and learning online. Cambridge (EE.UU.): Massachusetts Institute of Technology Press,1995)

- HERNÁNDEZ, P. (1997). Construyendo el constructivismo: criterios para su fundamentación y aplicación escolar. En RODRIGO, M ${ }^{\mathrm{a}}$.J. ARNAY, J. (Comps). La construcción del conocimiento escolar, Barcelona/Buenos Aires/México, Paidós, 285-312

- HEPP, P.(2003). Enlaces: El programa de informática educativa de la reforma educacional chilena. En COX, C. (Editor) Políticas educacionales en el cambio de siglo: La reforma del sistema escolar de Chile, Santiago, Editorial universitaria, 419-451

- MACDONALD, J. (2003). Assessing online collaborative learning: process and product. Computer \& Edutacion, 40 (4), 377-391.

- MUILENBURG, L..Y. y BERGE, Z.L. (2001). Barriers to distance education: A factor-analytic study. The American Journal of Distance Education, 15(2), 7-22. Disponible en: : http://www.emoderators.com/zberge/fa_ajde_050401.shtml [2003,Octubre 4]

- PÉREZ, A. (2002). Elementos para el análisis de la interacción educativa en los nuevos entornos de aprendizaje, pixel-bit revista de medios y educación [en línea], 19. Disponible en: http://www.sav.us.es/pixelbit/articulos/n19/n19art/art1904.htm [2003, Abril 1]

- RYAN, S., SCOTT, B., FREEMAN, H. y PATEL, D. (2000). The virtual university: the Internet and resoursce-based learning, London, Kogan Page.

- SALMON, G. (2000). E-moderating: The key to teaching and learning online, London: Kogan Page.

- SWAN, K., SHEA, P. FREDERICKSEN, E., PICKETT, A. PELZ, W. y MAHER, G. (2000). Building knowledge building communities: consistency, contact and communication in virtual classroom, Journal Educational Computing Research, 23 (4), 359-381.

- VIGOSTKY, L.S.(1978). El desarrollo de los procesos psicológicos superiores, Barcelona,Crítica. 
- TOLMIE, A. y BOYLE, J. (2000). Factors influencing the success of computer mediated communication (CMC) environments in university teaching: a review and case study, Computers $y$ Education, 34 (2), 119-140.

\section{NOTAS}

[1] La investigación lleva por titulo "Caracterización del rol del tutor en una entorno virtual de aprendizaje para la formación docente: curso a distancia funciones matemáticas en la enseñanza media. Es dirigida por la profesora Begoña Gros.

[2] Datos obtenidos de www.redenlaces.cl página oficial del proyecto Enlaces

[3] Proyecto de informática educativa del Ministerio de Educación de Chile, que provee a los establecimientos de computadores, acceso a Internet, capacitación en el uso de TIC y asistencia técnica.

[4] Resultados del estudio "Penetración y uso de las tecnología en los profesores" http://www.redenlaces.cl/documentos/informe.pdf1

[5] www.funciones.cl Sitio oficial del curso "Funciones matemáticas en la enseñanza media.

(C) Ediciones Universidad de Salamanca 